\title{
e-Phaïstos
}

e-Phaïstos

Revue d'histoire des techniques / Journal of the history

of technology

VII-1 | 2019

Le travail humain

\section{Les mesures du corps en activité et la concrétisation de l'homme}

Les apports de l'histoire des sciences du travail aux sciences humaines contemporaines

The Measurements of the Body in Activity and the Concretization of the Man. The Contributions of the History of the Sciences of Work to the Contemporary Human Sciences.

\section{Marco SARACENO}

\section{OpenEdition}

Journals

Édition électronique

URL : http://journals.openedition.org/ephaistos/4499

DOI : 10.4000/ephaistos.4499

ISSN : 2552-0741

Éditeur

IHMC - Institut d'histoire moderne et contemporaine (UMR 8066)

Référence électronique

Marco SARACENO, «Les mesures du corps en activité et la concrétisation de l'homme », e-Phaïstos [En ligne], VII-1 | 2019, mis en ligne le 06 avril 2019, consulté le 01 mai 2019. URL : http:// journals.openedition.org/ephaistos/4499; DOI : 10.4000/ephaistos.4499

Ce document a été généré automatiquement le 1 mai 2019.

Tous droits réservés 


\section{Les mesures du corps en activité et la concrétisation de l'homme}

Les apports de l'histoire des sciences du travail aux sciences humaines contemporaines

The Measurements of the Body in Activity and the Concretization of the Man. The Contributions of the History of the Sciences of Work to the Contemporary

Human Sciences.

Marco SARACENO

1 Ce n'est que relativement récemment que l'histoire des sciences a saisi «l'objet» "science du travail humain ${ }^{1}$, et ce, malgré le fait que le travail a été un objet fondateur des sciences humaines et sociales ${ }^{2}$. Si cela est dû en partie au rôle ancillaire de l'histoire des sciences de l'homme, cela est également dû à la difficulté de saisir un objet spécifique. Or, depuis le milieu du XIX ${ }^{\mathrm{e}}$ siècle, l'expression «science du travail» commence à être utilisée. On voit apparaître le terme «ergologie » vers la fin du XIX ${ }^{e}$ siècle (de manière dispersée entre la France, l'Italie, l'Allemagne et la Belgique), et, en 1904, une Revue de la science du travail est fondée en France. Mais cette ambition typique de la fin du XIX $X^{e}$ siècle de définition de nouveaux «domaines » de la science fera long feu, pendant que le terme ergonomie réussira à s'imposer dans l'après-guerre, non pas comme appellation d'une «science du travail», mais comme dénominateur commun de différentes techniques agissant sur les conditions de déroulement du travail. L'historien des sciences aurait donc bien du mal à retrouver dans ces quelques apparitions éparses de l'expression « science du travail » un objet de recherche.

2 Qu'est-ce que serait une science du travail ? Est-ce qu'il s'agirait d'une science censée découvrir l'objectivité d'un phénomène que l'on peut appeler «travail »? Ou est-ce qu'il s'agirait plutôt d'une science qui étudie les conditions d'utilisation de l'homme dans un contexte productif - faisant donc du travail non pas l'objet, mais le terrain d'application de la connaissance ? La difficulté à définir les bornes d'une histoire des sciences du travail est en ce sens évidente. Dans cette même perspective, la physiologie est-elle une science du travail parce qu'elle étudie le "travail physiologique», ou bien faut-il qu'elle soit 
appliquée à l'organisation de l'activité professionnelle pour rentrer dans cette catégorie ? D'autre part : la thermodynamique - définissant les mouvements organiques comme un «travail» au sens d'une transformation énergétique - et la psychologie appliquée à l'orientation professionnelle peuvent-elles être traitées comme deux aspects d'une même entreprise scientifique ? Cette dernière question renvoie à une tension constante dans la mobilisation du terme «travail » parmi les sciences de l'homme. Cette «notion de sens commun $»^{3}$ est en effet tantôt mobilisée pour rendre compte d'une "réalité » mécanique, physique, biologique, économique de la "nature humaine", tantôt évoquée pour renvoyer à un contexte social et technique d'utilisation de cette «nature ».

Cet article part du constat que cette difficulté à saisir une science du travail a conduit à des interprétations biaisées des entreprises scientifiques qui peuvent être classées sous cette bannière. En les lisant souvent comme des tentatives réductionnistes et explicitement orientées à "l'exploitation de l'homme par l'homme", on est souvent passé à côté de la richesse épistémologique de ces différentes tentatives de saisir scientifiquement le travail humain. Nous proposons donc de rentrer dans l'étude historique de ces tentatives scientifique non pas en les jugeant en fonction de leur capacité à saisir leur prétendu " objet ", le travail, lequel se révèle fuyant et conduit aux difficultés que nous avons évoquées, mais à partir de la définition de leurs "pré-textes ", selon la belle expression de Georges Canguilhem, c'est-à-dire des « raisons » qui guident l'entreprise d'objectivation. Ces raisons qui sont à la fois sociales et épistémologiques, peuvent être résumées dans la quête d'une mesure du corps en activité. En effet, les sciences du travail trouvent, dans l'activité productive, à la fois l'occasion de produire des normes universelles de gestion du corps qui les légitiment socialement et les conditions expérimentales pour étudier ce processus si original de "l'économie vitale " par laquelle un organisme «mesure» constamment son activité en fonction d'un contexte environnemental. Par cette entrée, nous montrerons que l'histoire de la « mesure du corps en activité " pose des questions épistémologiques riches d'enseignements pour les sciences humaines contemporaines, en quête de paradigmes épistémologiques permettant de dépasser les « grands partages » du passé.

\section{Les mesures du corps en activité, "pré-texte » de la science du travail}

Or, la difficulté de la science à définir un « objet-travail » a contribué à rendre complexe l'entreprise historique de caractérisation d'un «objet» «science du travail». Cela s'explique non pas parce que l'histoire des sciences aurait besoin pour se déployer de trouver derrière les méthodes et les discours une «entité réelle » qui en définirait les limites, mais plutôt parce que cette difficulté à saisir un « objet-travail » est l'expression d'une pluralité de "pré-textes $»^{4}$ que la science trouve dans l'objectivation du travail humain. En effet, comme le rappelle Georges Canguilhem, l'objet de l'histoire des sciences est tout à fait autre par rapport à l'objet de la science. Autrement dit: l'histoire des sciences ne s'intéresse pas aux conditions de connaissance d'un objet "initial " et «naturel » sur lequel agirait l'entreprise scientifique, mais elle s'intéresse à la manière dont la science construit son « objet ». Ainsi, l'objet « initial » de la science n'est qu'un "prétexte», et c'est en tant que tel qu'il doit être pensé par l'histoire des sciences. Le terme de « pré-texte » est ici pris au double sens d'une raison apparente qui dissimule un autre objectif, mais aussi d'un objet qui précède le travail de «textualisation» de la 
science, c'est-à-dire la production d'une "objectivation ». L'histoire des sciences du travail doit donc étudier à la fois l'objectif que la science cherche à concrétiser par l'objectivation du travail humain et les phénomènes variables qu'elle résume au fur et à mesure dans son objet-travail. Or, justement, le processus d'objectivation par lequel la science construit l'objet-travail-humain paraît extrêmement éclaté. La notion de travail sert à la fois (par exemple) à faire rentrer la physiologie et la psychologie dans le paradigme énergétiste et à légitimer la science positive comme support du gouvernement capitaliste ; à « objectiver » la "volonté » et à discipliner les mouvements corporels. De même encore, le «travail » sert à définir aussi bien les mouvements organiques que les actes intentionnels, les efforts, les prédispositions psychomotrices individuelles, etc.

Ce caractère fuyant de l'objet scientifique « travail » peut conduire ceux qui s'aventurent dans son historicisation à des interprétations partielles. On peut en effet penser que le «pré-texte » du travail soit l'activité productive du corps, au sens de l'agir intentionnel. Cela conduit à voir dans l'objectivation du travail le "prétexte " à un réductionnisme général de l'activité humaine aux fonctionnements mécaniques ou énergétiques de l'organisme ${ }^{5}$. D'un autre point de vue, on peut penser que «l'objet» qui précède l'entreprise scientifique d'objectivation du travail soit l'activité productive du corps, au sens d'une dépense mécanique. Cela conduit à penser la science du travail comme un "prétexte» pour "naturaliser» "l'invention $"^{6}$ du travail de la société capitaliste ${ }^{7}$. Toutefois ces approches empêchent de saisir l'ambivalence du terme "prétexte »: la première renvoie en effet à un objet-travail «naturel » qui préexisterait à la démarche d'objectivation et que celle-ci ne pourrait approcher que par de grossières approximations ; tandis que la deuxième considère que la construction d'un « objet scientifique travail» n'est que le prétexte pour la réalisation d'un objectif social ou idéologique.

6 Afin d'éviter ces lectures partielles, il convient d'éviter d'entrer dans l'histoire de la science $\mathrm{du}$ travail par les questions: "de quelle manière la science objective le "travail humain"? », ou " pourquoi la science objective-t-elle le travail ? "; mais plutôt en se demandant: "quelle démarche d'objectivation la science met en place pour la constitution d'un objet "travail humain" ?", ou "qu'est-ce que l'homme cherche à "objectiver" à travers la "science du travail humain" ?». Or, cela implique d'une part de s'intéresser aux "techniques» scientifiques, c'est-à-dire aux outils et aux pratiques que la science développe pour arriver à définir le «travail », et d'autre part d'inscrire l'histoire de la science du travail dans un questionnement plus large sur l'activité humaine d'objectivation. Cela conduit alors à inscrire l'histoire de l'objet scientifique «travail» à la fois dans une histoire générale de la technicité et dans l'histoire épistémologique des sciences humaines et sociales.

7 C'est en ce sens que l'étude des mesures du corps en activité se révèle une entrée particulièrement heuristique pour définir l'objet « science du travail ». En effet, le corps en tant que support à la fois matériel et subjectif de l'activité par laquelle l'homme « objective » sa nature dans le monde - et la mesure - en tant qu'outil de modélisation, mais aussi de gestion de l'activité - permettent de saisir le double sens du terme " prétexte » évoqué plus haut. L'engagement corporel dans une activité proportionnée à un but est en effet le prétexte de toute objectivation du travail: c'est cette utilisation mesurée des «forces humaines » par l'homme qui représente « l'objet » (ante litteram) de la science du travail. Et, en même temps, la mesure de l'activité du corps selon un étalon 
établi par convention est le prétexte pour intégrer l'homme comme « objet » d'un projet de connaissance et de gestion « rationnel ».

Dans cette perspective, l'histoire des mesures par lesquelles on a cherché à connaître « l'activité humaine organisée avec mesure » se révèle un outil extrêmement puissant de réflexivité pour les sciences humaines contemporaines, engagées depuis trois décennies dans une refonte impliquant de prendre en compte la place de leur propre démarche scientifique dans le processus humain d'objectivation ${ }^{8}$. Notre article se propose donc d'évoquer la manière dont le travail historique sur la « science du travail » - en suivant les tâtonnements par lesquels l'homme ne cesse " d'objectiver» (au sens de «rendre concret») son potentiel d'activité dans une continuité entre biologique, sociologique et existentiel - permet d'éclairer certaines orientations contemporaines de l'épistémologie des sciences humaines.

\section{Instrumentalisation et spontanéité}

Si les sciences humaines et sociales ont progressivement intégré à leur recherche la dimension corporelle de l'activité, c'est à travers la prise en compte de la pluralité des mobilisations du corps dans la vie humaine - des mobilisations qui ne se limitent pas à la simple activation d'un système d'organes. Dans cette perspective, la façon d'aborder la question du corps en sciences humaines et sociales a été largement marquée par la fameuse formule de Marcel Mauss, définissant le corps comme « premier instrument » de l'homme'. La définition des techniques du corps permet en effet de voir dans les plus différents usages du corps la trace matérielle de valeurs et normes constamment négociées et « incorporées ».

10 Toutefois, la prise en compte de cette « instrumentalisation » du corps servait à penser l'homme dans sa « totalité », et donc à " unifier » la dimension corporelle de l'action aux dimensions psychologique et sociale. Au-delà de l'expression d'«homme total» (probablement malheureuse), cela renvoyait à la nécessité d'éviter de penser l'activité humaine soit comme une manifestation purement culturelle déliée des conditions matérielles de sa réalisation, soit comme la simple utilisation d'éléments matériels, lesquels détermineraient par leurs qualités «naturelles» le déroulement de l'activité. L'approche de Mauss ne cherchait donc pas à soustraire le «corps » aux sciences de la «nature » pour le faire devenir un «objet» des sciences de l'homme : l'anthropologue trouvait au contraire dans la question du corps comme « instrument » la démonstration de la nécessité de dépasser cette distinction même.

11 Toutefois, si le corps a fait de plus en plus l'objet de recherches en sciences humaines, l'assomption du programme de l'anthropologie maussiènne est-elle fort relative ${ }^{10}$. Les sciences humaines et sociales assument ainsi le corps soit comme le support de phénomènes sociaux - et donc comme un "objet miroir» permettant d'accéder au «véritable " fait social -, soit comme un ensemble de mécanismes matériels rendant compte de l'ancrage «naturel» de certains phénomènes sociaux. Par-delà, l'idée forte d'« instrumentalisation » - par laquelle Mauss cherchait justement à montrer que dans l'activité humaine les contraintes matérielles, psychologiques et sociales se confondent n'est souvent mobilisée que de manière partielle par les sciences humaines contemporaines qui se penchent sur l'objet « corps ». 

instrument est pensée comme une exploitation ou un asservissement dans une perspective qui a largement influencé les recherches sociologiques sur le travail ${ }^{11}$. La réflexion sur le corps «instrument de l'homme» est donc inscrite dans une interprétation de la «technique " d'inspiration heideggérienne comme occultation de «l'authenticité humaine ». Cette lecture de "l'instrumentalisation du corps $»^{12}$ comme «domination» et «moralisation» de l'être-homme a pu se répandre dans les sciences sociales grâce au succès des catégories bourdieusiennes et foucaldiennes d'« habitus » et de «disciplinarisation». D'autre part, le corps «premier instrument» a été pensé à travers (notamment) les travaux de psychologues tels que Vygotski comme le fondement de l'activité humaine "d'instrumentation", c'est-à-dire de la capacité à produire des schémas d'activité. Dans la mesure où l'homme se sert de son corps de manière instrumentale, il sait trouver des solutions pratiques ${ }^{13}$. Or, si la première de ces deux perspectives semble confondre l'instrumentalisation technique du corps avec son asservissement aux normes sociales, la seconde ne semble prendre en compte que de loin l'impact de ces normes sur les usages techniques des corps ${ }^{14}$. Une histoire des sciences du travail attentive aux contextes de son déploiement - et qui ne chercherait donc dans cette tradition $n i$ un modèle épistémologique de définition du corps, $n i$ l'expression d'une volonté exogène de domination - pourrait contribuer à saisir la complexité de la question de « l'instrumentalisation » du corps dans la perspective jadis ouverte par Mauss.

L'historicité des techniques de mesure de la science du travail - c'est-à-dire des mesures socialement intelligibles de l'usage mesuré du corps par le corps - montre l'impossibilité de faire la part entre ce qui, dans l'action, dépend de la « technicité naturelle » du corps et ce qui dépend du façonnage social - auquel d'ailleurs la science elle-même contribuerait. L'évolution historique des mesures des sciences du travail apparaît dès lors soit comme le résultat des modifications des besoins sociaux d'usage des corps (qui conduisent à mettre l'accent sur des aspects différents du fonctionnement du corps), soit comme l'effet d'une transformation des usages psychomoteurs du corps lui-même (qui évoluent à cause de la transformation des cadres sociaux d'organisation de l'activité). C'est là un des enseignements les plus importants de l'histoire de la mesure de la fatigue au travail. Cette histoire montre que, pour s'approcher d'une « objectivation » de l'économie par laquelle le corps « utilise ses forces ", il faut prendre en compte les usages socio-économiques du corps qui - par l'apparition de facteurs psychosociologiques (monotonie, niveau salarial, etc.) - modifient la perception subjective de l'effort ${ }^{15}$. L'histoire des sciences du travail et de leur relation ambivalente avec la mesure permet donc de ne pas opposer ces deux aspects de l'instrumentalisation du corps, mais au contraire d'en saisir la complémentarité.

L'histoire de la science du travail contribue donc à une réflexion épistémologique sur la place du corps dans le discours des sciences humaines et sociales. Elle contribue également à réfléchir à la mesure comme instrument pratique, telle qu'elle est saisie par des nombreuses recherches en sciences humaines et sociales. En effet, depuis les travaux séminaux de Kula, les mesures et leurs usages sont devenus un objet à part entière pour les sciences humaines et sociales ${ }^{16}$. Ces recherches se sont concentrées notamment sur la pluralité des métrologies et sur leur ancrage dans les pratiques sociales et culturelles. Cela a permis d'éviter d'aborder la mesure comme un instrument neutre capable de décrire un fantomatique "réel», pour s'intéresser au contraire aux débats et aux «conventions» qui déterminent l'apparition et l'utilisation d'une mesure. Ainsi la 
mesure a-t-elle été étudiée à la fois comme un instrument de la "gouvernementalité " par laquelle une " autorité sociale » se donne les moyens de " conduire les conduites ${ }^{17}$, et comme une pratique à part entière - une pratique dans laquelle les acteurs s'engagent et par laquelle ils cherchent à réaliser des intérêts et des valeurs contrastants ${ }^{18}$. Ces deux perspectives ne sont pas à opposer, en pensant d'un côté une critique sociale de la mesure et, d'autre part, une « essentialisation » de la mesure.

Or l'histoire des sciences du travail montre justement que, si la mesure est un outil par lequel une autorité conduit les sujets selon des normes exogènes à leur activité, c'est parce qu'elle contribue à la production et à la légitimation de valeurs socialement établies. De même, si la mesure devient une ressource pour l'activité individuelle, c'est parce qu'elle permet d'inscrire l'activité du sujet dans un espace commun partagé. L'histoire des mesures du corps en activité montre ainsi une circularité constante : la science a produit des «mesures » pour «normaliser » les régulations «spontanées » du corps; or, elle contribue ainsi à façonner des manières nouvelles de se servir du corps, et donc de nouvelles «mesures spontanées» qui deviendront de nouveaux objets à " mesurer».

16 C'est par exemple ce que montre l'histoire de la mesure du rendement. Si c'est en vue de « discipliner» les travailleurs que l'on cherche à mesurer le rendement "moyen », cela conduit toutefois à de nouvelles formes de "gestion de soi ", c'est-à-dire à de nouvelles mesures de son propre effort dans ce que l'on a pu appeler le « freinage ». Les ouvriers réduisent volontairement leur effort, non pas pour se reposer, mais pour reprendre " affectivement » le " contrôle » de leur activité. Or, comme l'a montré Max Weber, cela impliquait de repenser les mesures de l'effort en introduisant le rôle de l'affectif et du «subjectif $»^{19}$. De plus, la connaissance des mesures du rendement sert aux travailleurs eux-mêmes pour "freiner » de manière plus « rationnelle ", ainsi que l'a montré Donald Roy $^{20}$. Cela permet de mettre l'accent sur les rapports sans cesse transformés entre les prétendues «mesures spontanées » (par lesquelles le sujet évalue la portée de son action) et les « mesures institutionnalisées » cherchant à rationaliser cette action ${ }^{21}$.

\section{Normativité et subjectivité}

Or la confrontation entre un « corps » objectivé par la science (composé d'une matérialité «mesurable») et un «corps-sujet» (siège premier de l'intentionnalité) est un topos classique de la philosophie contemporaine, au moins depuis la réflexion phénoménologique. Cette approche a joué un rôle essentiel dans les travaux sur l'éthique médicale, en permettant de saisir la continuité et la différence entre le corps vu comme l'incarnation d'une histoire singulière et le corps vu comme une donnée physiologique sur laquelle agir de manière «normalisée $»^{22}$. De plus, la prise en compte de la corporéité de l'activité a fourni à la philosophie des outils pour retrouver dans l'activité humaine des ressources d'émancipation subjective par rapport à l'ordre social capitaliste. Ainsi, en pensant le corps comme «machine désirante $»^{23}$, des auteurs comme Deleuze - et, de manière différente, Foucault - ont retrouvé dans l'incorporation de la subjectivité un pouvoir émancipateur vis-à-vis des normes qui cherchent à le « contrôler ".

18 Ces lectures ont insisté sur l'importance de distinguer le corps comme support de la subjectivité et le "corps-organique », défini par les «lois » causales déterminant son fonctionnement. La prise en compte d'un corps-sujet semblait en effet nécessaire au dépassement du couple «corps-esprit» de la philosophie cartésienne. Toutefois, ces 
approches rendaient inconciliable le corps "objectivé » de la science - qui apparaît comme un simulacre "produit " pour mieux normaliser le corps dans sa "réalité " historique et somatique - et le corps comme siège de siège de l'activité créatrice de sens. Dans cette perspective, l'activité du corps paraît destinée à être pensée soit comme l'un des résultats de la normalisation du corps sujet, soit comme l'expression d'une force qui échappe à toute objectivation. Autrement dit : l'opposition entre corps «normalisé » et corps « sujet » ne permet pas de saisir l'activité technique telle que nous l'avons évoquée plus haut.

Or, l'histoire de la science du travail montre justement que c'est en définissant les normes du corps-mécanisme (c'est-à-dire du corps pensé comme un engin fonctionnant selon des lois universelles dont la science et dont l'activité sont donc modélisables) que l'on produit les conditions de l'apparition d'un corps support de choix (de valeurs, d'engagements subjectifs) et créateur de "faits nouveaux ». Le corps ne peut produire des "événements nouveaux » que parce qu'il oriente son activité «mécanique ». La définition de normes "physiologiques ", "énergétiques» ou encore "mécaniques» de l'activité corporelle fournit donc des occasions d'activité. Or, cela ne veut pas dire que ces normes seraient la simple constatation du fonctionnement naturel du corps ; au contraire, elles sont toujours orientées par des valeurs et des attentes sociales. En définissant le corps d'une certaine manière, la science contribue donc à le façonner, mais ce façonnement est également l'occasion de la concrétisation d'un sujet. Par exemple, l'établissement de critères précis de sélection de la main-d'œuvre à partir de tests psychotechniques est indéniablement une façon de normaliser les choix individuels afin de répondre aux exigences de la production économique ${ }^{24}$; mais la quête de mesures pour rationaliser le marché du travail se révèle également une condition pour permettre la rencontre entre les intérêts subjectifs et les valeurs sociales ${ }^{25}$. C'est dans cette perspective d'ailleurs que la psychotechnique a largement contribué, avec ses mesures des "aptitudes", aux évolutions pédagogiques de l'école «nouvelle ».

20 Comme nous l'a en effet appris la philosophie du vivant de Georges Canguilhem, l'activité vitale implique une renormalisation incessante de sa relation avec le milieu. Cela signifie que le corps-objet de l'homme transforme constamment son fonctionnement afin de répondre aux exigences nouvelles que son milieu lui impose. Or, ce milieu est justement le milieu social dans lequel le corps est façonné pour être plus « efficace » d'un point de vue productif. S'esquisse alors une circularité qui conduit à penser le corps produit des normes comme une continuation du corps producteur de normes. L'engagement «subjectif» du corps implique toujours une incorporation de normes «objectives». Ainsi, la mesure normative du corps en activité - en tant qu'évaluation de "l'activité optimale » du corps - ne serait rien d'autre que la continuation (à un niveau collectif) de l'autorégulation du corps en activité. La mesure du corps en activité de la science du travail n'est - en ce sens - que la continuation de l'activité mesurée du corps, de la même manière que pour Georges Canguilhem la connaissance de la vie n'était que sa continuation typiquement $«$ humaine $»^{26}$.

21 En partant de la mesure du corps en activité comme pré-texte de la science du travail c'est-à-dire en partant de la manière dont une structure "objectivement mesurable » conçoit constamment des nouvelles façons de «s'utiliser avec mesure »-, l'histoire des sciences du travail contribue à explorer la complexité anthropologique de la norme. Mesurer, c'est à la fois une manière de saisir les pratiques en les réduisant à un modèle normatif «standard» et une façon de produire de nouvelles normes fonctionnelles à 
l'activité poursuivie. Ce que montre l'histoire des sciences du travail, ce sont des allersretours constants entre la prise en compte de la variabilité historique de l'engagement subjectif du corps en activité et la nécessité de fournir des normes «objectives » pour permettre à cet engagement de se concrétiser dans des objectifs valorisés socialement. L'histoire de la science du travail montre la difficulté - rencontrée par les démarches ayant cherché à mesurer le corps en activité - à faire la part entre plusieurs normes " antécédentes " ${ }^{27}$ (les normes sociales que les mesures elles-mêmes véhiculent, les normes organiques qu'elles voudraient objectiver, et les évaluations subjectives qui transforment constamment l'activité du corps). En ce sens, elle contribue à penser le corps comme un carrefour de " débats de normes ", et donc comme le moment où la libre subjectivité et la contrainte normative "antécédente » (qu'elle soit physiologique ou sociale) se croisent et s'alimentent réciproquement.

L'histoire des sciences du travail permet donc de chercher derrière les pratiques métrologiques les solutions provisoires et toujours en tension par lesquelles le corps est utilisé par des sujets inscrits dans des ordres sociaux avec leur «normativité » propre. Comme l'écrit le médecin français Armand Imbert ${ }^{28}$, acteur de premier plan dans la constitution de la science du travail, l'homme est le meilleur physiologiste, puisqu'il est la physiologie elle-même ; mais cela ne signifie pas que le travail métrologique de la science n'ait pas d'intérêt. Au contraire, il sert aux hommes en sociétés à mieux confronter leurs « expériences » du corps afin de les inscrire dans un horizon commun.

De ce point de vue, l'histoire des sciences du travail permet de sortir d'une épistémologie binaire de l'étude du corps en activité: un discours qui opposerait une "sortie du laboratoire » attentive au travail « réel » (au sens de l'expérience contingente et vécue du corps en activité) à une modélisation « hors-sol » d'un corps réduit à du mécanisme. Cette posture a sans aucun doute servi à l'évolution épistémologique des sciences du travail on pense notamment à l'ergonomie du deuxième après-guerre - en permettant de prendre du recul sur la limite intrinsèque des modélisations de l'activité du corps. Toutefois, lorsque l'on regarde historiquement cette distinction, il apparaît clairement que la " sortie du laboratoire » n'est pas la conquête d'un savoir finalement éclairé, mais le topos qui accompagne depuis les premières recherches sur le "travail humain » toute production de nouvelles modélisations.

C'est en ce sens que les travaux sur l'histoire des sciences du travail contribuent au renouveau « pragmatiste » des sciences humaines et sociales. Ce n'est pas ici le lieu pour discuter de la pertinence de cette idée de «tournant pragmatiste ». Comme on le sait, dans les trois dernières décennies, la réception de l'approche pragmatiste est plurielle; on peut néanmoins considérer que ce tournant consiste généralement à fonder l'épistémologie des sciences humaines et sociales sur l'idée d'une "boucle de l'enquête ${ }^{29}$ - Cela implique de penser les sujets comme des acteurs qui interagissent avec leur environnement en évaluant et en expérimentant les conditions de leur activité. Or cela entraîne deux conséquences. D'une part, cela fait des sciences humaines des sciences de l'enquête, c'est-à-dire des sciences engagées à suivre les stratégies d'évaluation du réel mobilisée par les acteurs (en évitant donc d'interpréter leurs activités à partir de leurs déterminants sociaux ou d'un dispositif général d'optimisation). D'autre part, cela implique que la science elle-même contribue à l'avancée d'un travail d'enquête collectif, démocratique, dans lequel la société cherche à déterminer les orientations et les conditions de succès de l'activité humaine. Faire de l'histoire des sciences du travail implique justement de suivre les mesures de l'activité du corps comme autant de 
tentatives de connaissance des stratégies corporelles de production d'une activité efficace - une connaissance par laquelle la science contribue au débat social sur l'idée même d'efficacité.

\section{Conclusion}

En étudiant la transformation des pré-textes d'une science du travail - c'est-à-dire des mesures du corps en activité (au sens à la fois des façons dont l'homme utilise son corps avec mesure et des normes standard produites pour utiliser le corps socialement) l'histoire des sciences du travail se révèle un puissant antidote contre toute lecture partiale des renouveaux qu'ont connu les sciences humaines et sociales dans les dernières décennies. Ces renouveaux impliquent en effet d'abandonner les «partages » classiques entre « nature et culture ", "sujet et objet » ou " connaissance et expérience » - afin de bâtir un savoir attentif aux relations entre ces différentes composantes de l'agir humain. En montrant l'impossibilité de séparer les deux sens de son pré-texte (c'est-à-dire des " mesures du corps en activité »), l'histoire de la science du travail est un fort rappel qui montre que dans l'étude de l'activité humaine, toute analyse dichotomique cherchant à différencier "l'authentique », "le réel » et "l'incommensurable» de "l'artificiel», «l'instrumental» et le «quantifiable » signifie mécomprendre l'activité par laquelle l'homme s'objective dans l'homme et sur laquelle bâtir une science de l'homme.

\section{NOTES}

1. Un des textes fondateurs de ce domaine de recherche est RABINBACH Anson, The Human Motor: Energy, Fatigue and the Origins of Modernity, University of California Press, 1992. Dans le contexte français, on peut rappeler les travaux séminaux de Georges RIBEILL ( « Les débuts de l'ergonomie en France à la veille de la Première Guerre mondiale ", Le Mouvement social, 1980, nº113, p.3-36) et de Michel VALENTIN (Travail des hommes et savants oubliés. Histoire de la médecine du travail, de la sécurité et de l'ergonomie, Éd. Docis, Paris, 1978).

2. PILLON Thierry et VATIN François, Traité de sociologie du travail, $2^{\mathrm{e}}$ éd. actualisée, Toulouse, Octarès, 2007.

3. VATIN François, Le travail, sciences et société : essais d'épistémologie et de sociologie du travail, Bruxelles, Éd. de l'Université de Bruxelles, 1999.

4. «L'objet en histoire des sciences n'a rien de commun avec l'objet de la science. L'objet scientifique, constitué par le discours méthodique, est second, bien que non dérivé, par rapport à l'objet naturel, initial, et qu'on dirait volontiers, en jouant sur le sens, pré-texte. L'histoire des sciences s'exerce sur ces objets seconds, non naturels, culturels, mais n'en dérive pas plus que ceux-ci ne dérivent des premiers. [...] L'objet du discours historique est, en effet, l'historicité du discours scientifique, en tant que cette historicité représente l'effectuation d'un projet intérieurement normé ", CANGUILHEM Georges, Études d'histoire et de philosophie des sciences, Paris, J. Vrin, 1968, p.17. 
5. C'est ainsi que la psychologie du travail contemporaine a souvent refoulé son passé historique « réductionniste ». Pour une analyse critique de cette histoire " présentiste », voir CLOT Yves, Les histoires de la psychologie du travail : approche pluri-disciplinaire, Toulouse, Octarès, 1996.

6. MÉDA Dominique, Le travail : une valeur en voie de disparition, Paris, Aubier, 1996.

7. Celle-ci est par exemple une des interprétations de RABINBACH, The Human Motor..., 1992, op. cit.

8. DOSSE François, L'Empire du sens: l'humanisation des sciences humaines, Paris, La Découverte/Poche, 1998.

9. MAUSS Marcel, «Les techniques du corps », Journal de Psychologie, XXXII, 3-4, 1936.

10. On reprend ici des thématiques largement développées par BERTHELOT Jean-Michel, «Du corps comme opérateur discursif, ou les apories d'une sociologie du corps ", Sociologie et sociétés, 24, 1992.

11. PILLON Thierry, «De quelques traces du corps en sociologie du travail », Sociologie et sociétés, 2016, vol. 48, $\mathrm{n}^{\circ} 1, \mathrm{p} .83$.

12. Voir par exemple GLEYSE Jacques, L'instrumentalisation du corps : Une archéologie de la rationalisation instrumentale du corps, de l'âge classique à l'époque hypermoderne, Paris, Éditions L'Harmattan, 2000.

13. RABARDEL Pierre, Les hommes et les technologies; approche cognitive des instruments contemporains, Paris, Armand Colin, p.239, 1995.

14. C'est à une conclusion similaire qu'arrive Georges Vigarello dans la postface qu'il a écrit pour la dernière édition de son ouvrage Le corps redressé dans laquelle il inscrit son travail dans le contexte des sciences humaines sociales des années 1970. VIGARELLO Georges, Le corps redressé: histoire d'un pouvoir pédagogique. Nouvelle édition avec postface, Paris, A. Colin, 2004.

15. SARACENO Marco, "L'expérimentation instrumentale et l'instrumentalisation de l'expérience. Succès et déclin de l'ergographe dans la représentation de l'expérience quotidienne du travail », in G. FATET (dir.), Rôle et fonction de l'instrument, PULIM, 2016, p.107-132. Voir aussi SARACENO Marco, " La fatica come misura del lavoro utile ", Rivista di storia dell'Università di Torino, V. III, $\mathrm{n}^{\circ} 1,2014, \mathrm{p} .69-84$.

16. WITOLD Kula, Les mesures et les hommes, Paris, Éd. de la MSH, (1970), 1984.

17. DESROSIÈRES Alain, La politique des grands nombres. Histoire de la raison statistique, Paris, La Découverte, 2000.

18. BIDET Alexandra et VATIN François, "Acteur et mesure au travail », Traité de sociologie économique, Paris, PUF, $2^{\mathrm{e}}$ édition, 2014, p.711-747.

19. WEBER Max, Zur Psychophysik der industriellen Arbeit: Schriften und Reden 1908-1912, Tübingen, J.C.B. Mohr (P. Siebeck), 1995. Voir notre article SARACENO Marco, «La razionalità incorporata : l'analisi psico-fisiologica del lavoro industriale nell'opera di Max Weber », in G. SOLANO, F. SOZZI (dir.), Maestri delle scienze sociali, LiminaMentis Editore, 2012, p.349-370.

20. ROY Donald, «Deux formes de freinage dans un atelier de mécanique : respecter les quotas et tirer au flanc. Précédé d'une présentation de J.P Briand et J.M Chapoulie », Sociétés Contemporaines , 2000, vol.40, nº1, p.29-56.

21. On suivra dans cela l'idée d'économie spontanée développée par le « Groupe Mesure » autour de François Vatin : François Vatin, Gwenaële Rot, Camille Palazzo, Odette Minh Fleury, Manuel Boutet, Alexandra Bidet et Thomas Le Bianic, «Le sens de la mesure. Manifeste pour l'économie en sociologie: usage de soi, rationalisation et esthétique au travail ", in Evaluer et Valoriser. Une sociologie économique de la mesure, Toulouse, Édition du Mirail, 2013.

22. On renvoie ici à la distinction entre "corps donné " et "corps produit » esquissée par Georges CANGUILHEM dans ses Écrits sur la Médecine, Paris, Seuil, 2002.

23. DELEUZE Gilles et GUATTARI Félix, L'Anti-CEdipe. Capitalisme et schizophrénie, Paris, Éditions de Minuit. 1972

24. NAVILLE Pierre, Théorie de l'orientation professionnelle, Paris, Gallimard, 1945. 
25. Voir à ce propos la multiplicité des conceptions de la notion d'aptitude qui émergent au sein de la psychotechnique : SARACENO Marco, «L'école française de psychotechnique et la mesure scientifique de la valeur professionnelle ", in GUTIERREZ Laurent, MARTIN Jérôme et OUVRIERBONNAZ Régis (dir.), Henri Piéron (1881-1964). Psychologie, orientation et éducation, Octarès, 2016.

26. CANGUILHEM Georges, «Le concept et la vie », Revue Philosophique de Louvain, 1966, t. LXIV, n 82, p.193-223.

27. SCHWARTZ Yves et DURRIVE Louis, L'activité en dialogues : entretiens sur l'activité humaine (II), Toulouse, Octarès, 2009.

28. IMBERT Armand, «Rapport de la quatrième section: hygiène industrielle et hygiène professionnelle. Réponse à la troisième question", in Congrès international d'hygiène et de démographie, Bruxelles, Wessenbruch, 1903, p.1-16. Sur cet auteur, voir LE BIANIC Thomas et VATIN François, « Armand Imbert (1850-1922), la science du travail et la paix sociale », Travail et Emploi, 2007, no 111, p. 7-19. Voir également SARACENO Marco, «Mesurer le risque par la fatigue : la psychophysiologie dans les débats sur l'indemnisation des accidents de travail à la fin $\mathrm{du} \mathrm{XIX}^{\mathrm{e}}$ siècle", in Thomas LE ROUX (dir.), Risques industriels. Savoirs, régulations, politiques d'assistance, fin XVII - début XX'e siècle, PUR, 2016.

29. CEFAÏ Daniel, BIDET Alexandra, STAVO-DEBAUGE Joan, FREGA Roberto, HENNION Antoine et TERZI Cédric, "Introduction du dossier "Pragmatisme et sciences sociales: explorations, enquêtes, expérimentations" », SociologieS, 2015.

\section{RÉSUMÉS}

En partant de la question de la mesure du corps au travail, c'est-à-dire de la façon dans laquelle une structure "objectivement mesurable" conçoit constamment de nouvelles manières de "s'utiliser avec mesure », cet article se propose d'ouvrir une réflexion sur la place de la mesure du corps dans le discours des sciences humaines et sciales abordant l'activité technique. Les sciences humaines et sociales ont en effet trouvé dans la mesure un «objet technique» ambivalent. La mesure est à la fois un instrument technique pour agir sur le réel et un vecteur «d'instrumentalisation» du réel, par lequel un phénomène est "réduit » à une dimension "utile». La mesure du corps en activité a ainsi été conçue soit dans une perspective critique comme un outil visant à transformer le corps en un instrument docile au service de la production et de l'ordre social capitalistes; soit, selon une conception inspirée par la psychologie de l'activité comme la production spontanée du sujet qui cherche à utiliser de manière efficace ses schémas psychomoteurs en les adaptant à la situation contingente. L'histoire des techniques permet d'éviter d'opposer de manière dichotomique ces lectures, montrant derrière les mesures du corps au travail les solutions provisoires et toujours en tension par lesquelles le corps a été utilisé au jour le jour par des sujets inscrits dans des ordres sociaux.

Starting from the question of the measurement of the body at work, that is to say of the way in which an "objectively measurable" structure constantly designs new ways of "using with measure", this article proposes to open a reflection on the place of the measurement of the body in the discourse of the human and social sciences addressing the technical activity. Indeed, the human and social sciences have found an ambivalent "technical object" in the measure. Measurement is both a technical instrument for acting on the real and a vector of "instrumentalization" of the real, by which a phenomenon is "reduced" to a "useful" dimension. 
The measurement of the active body has thus been conceived from a critical perspective as a tool aimed at transforming the body into a docile instrument at the service of capitalist production and social order; or, according to a conception inspired by the psychology of activity as the spontaneous production of the subject who seeks to use his psychomotor schemas effectively and adapting them to the contingent situation. The history of techniques makes it possible to avoid dichotomously contrasting these readings, showing behind the measurements of the body at work the temporary and always-in-tension solutions by which the body has been used day by day by subjects enrolled in social orders.

\section{INDEX}

Mots-clés : histoire des techniques, XXe siècle, Europe, épistémologie, instrumentalisation, psychophysiologie

Index chronologique : Époque contemporaine Index géographique : Europe

Keywords : history of technology, XXth century, Europe, epistemology, instrumentalization, psychophysiology

\section{AUTEUR}

\section{MARCO SARACENO}

Docteur en Sociologie et Histoire des Sciences, Marco Saraceno est chercheur post-doctorat au CETCOPRA, Université Paris 1 Panthéon-Sorbonne. Il s'intéresse à l'histoire de la psychophysiologie de l'activité motrice avec une attention particulière à l'évolution de ses techniques de mesure et à sa relation avec les autres sciences humaines. Dans ce contexte, il a étudié l'histoire de la notion de fatigue en observant le rôle qu'elle joue durant le « moment 1900 » dans la « fondation » des sciences sociales et dans la rhétorique décadentiste de l'époque. Il travaille actuellement sur la socio-histoire des capteurs de l'activité physique et sur l'évolution de leurs techniques d'enregistrement graphique. 